\title{
Second-order sexual conditioning in male Japanese quail (Coturnix japonica)
}

\author{
LAWRENCE L. CRAWFORD and MICHAEL DOMJAN \\ University of Texas, Austin, Texas
}

\begin{abstract}
Second-order conditioning of social approach to a female conspecific in male Japanese quail was investigated in four experiments. Subjects that received paired first- and second-order trials acquired second-order conditioning in both Experiments 1 and 2 . In contrast, subjects that received paired first-order but unpaired second-order trials, and subjects that received unpaired first-order but paired second-order trials, did not acquire second-order conditioning. In Experiment 3 , subjects for whom the first-order conditioned stimulus was presented in extinction showed second-order conditioning comparable to that shown by subjects in a control group that did not receive the extinction procedure. In Experiment 4, subjects approached a second-order stimulus less when sexually satiated than when sexually deprived. These findings suggest that second-order sexual conditioning in quail is mediated by an association of the second-order stimulus with a representation of the unconditioned stimulus.
\end{abstract}

Second-order conditioning has been of considerable historical interest because it putatively increases the range of situations in which Pavlovian conditioning may occur. First-order Pavlovian conditioning requires the pairing of a conditioned stimulus (CS) with an unconditioned stimulus (US), such as food, water, or shock, that is biologically important to the subject. This limits first-order conditioning to situations in which subjects encounter unconditioned stimuli. In contrast, in second-order conditioning a previously conditioned stimulus (CS1) is used in the role of a US to produce conditioning to a new stimulus (CS2). Therefore, second-order conditioning can occur in situations that do not involve exposure to an unconditioned stimulus, provided that the subjects previously receive first-order conditioning.

Most modern studies of second-order conditioning have involved fear conditioning in rats (e.g., Rizley \& Rescorla, 1972) or appetitive conditioning in rats and pigeons (e.g., Holland \& Rescorla, 1975b; Rescorla, 1979). The present experiments were conducted to extend the study of secondorder conditioning to sexual behavior.

Second-order sexual conditioning was previously demonstrated in rats by Zamble, Hadad, Mitchell, and Cutmore (1985), but they measured conditioning only indirectly by demonstrating shorter ejaculation latencies

The research was supported by National Research Service Award MH09988 to L.L.C. and National Institute of Mental Health Grant MH39940 to M.D. L.L.C. is currently at the Department of Psychology, University of Georgia, Athens, GA 30602. Correspondence may be addressed to L. L. Crawford at the University of Georgia or to M. Domjan, Department of Psychology, University of Texas, Austin, TX 78712 (e-mail: domjan@psyvax.psy.utexas.edu). following exposure to the second-order stimulus. Sexual conditioning has been investigated more extensively in male Japanese quail (see Domjan, 1994, for a review). Studies with Japanese quail have confirmed that, as in rats, sexually conditioned stimuli can facilitate copulatory behavior (e.g., Domjan, Greene, \& North, 1989). In addition, these experiments have identified circumstances in which first-order conditioned stimuli can also directly elicit various conditioned responses (e.g., Akins, Domjan, \& Gutiérrez, 1994).

Male quail approach and remain near visible but physically inaccessible females (Domjan \& Hall, 1986) and also come to approach and remain near visual stimuli that have been paired with exposure to females (Crawford \& Domjan, 1993; Holloway \& Domjan, 1993a). The present experiments were conducted to determine whether approach behavior can also become conditioned to a secondorder CS. First-order conditioning was conducted by periodically opening a window that provided visual access to a female quail. The auditory and visual cues of the window being opened constituted CS1, and exposure to the female quail provided the US. Second-order conditioning was conducted by pairing a light (CS2) with opening of the window without visual exposure to the female.

Conditioned behavior reflects second-order conditioning if the behavior depends on both pairings of the firstorder CS (CS1) with the US and pairings of the secondorder CS (CS2) with CS1. The role of second-order pairings was examined in Experiment 1 . The role of firstorder pairings was examined in Experiment 2. The final two experiments were conducted to test the effects on second-order conditioned responding of various postconditioning manipulations that were introduced to elucidate the contents of second-order conditioning. The effect of extinguishing responding to the first-order CS was 
examined in Experiment 3. The effect of US devaluation was examined in Experiment 4.

\section{EXPERIMENT 1}

The necessity of CS1-CS2 pairings during secondorder trials was examined in Experiment 1. All subjects were given paired first-order trials. One group of subjects was then given paired second-order trials, and another group was given unpaired second-order trials.

\section{Method}

Subjects. Twenty-four adult male Japanese quail (Coturnix japonica), 3-5 months old at the start of the experiment, served as subjects. All had previously participated in an experiment on copulation with taxidermic models in a substantially different apparatus than that employed in the present experiment (Crawford, Akins, \& Domjan, 1994). Twelve adult female Japanese quail served as stimulus females and copulation partners. All the females were sexually receptive and were active egg layers.

The birds were hatched from eggs obtained from a randomly bred colony maintained at the University of Texas at Austin and were raised in mixed-sex brooders until they were separated by sex at 30-32 days of age, which is prior to sexual maturity. At this time, males were individually housed to prevent aggressive and sexual interactions between them. All birds had ad-lib access to food and water and were housed under a 16:8-h light:dark photoperiod (lights on at $0600 \mathrm{~h}$ and off at $2200 \mathrm{~h}$ ) to maintain them in reproductive condition.

Apparatus. Six $91 \times 122 \times 117 \mathrm{~cm}$ test arenas were used for training, as in Crawford and Domjan (1993). One wall, made of $2.5-\mathrm{cm}$ wire poultry mesh, was hinged on one side to permit access to the arena for routine cleaning and maintenance. The other walls were wooden and painted white. The floor was made of $1.3-\mathrm{cm}$ wire mesh. A $15.5 \times 18 \mathrm{~cm}$ observation window, centered along one wall at floor level, allowed the male to see a female quail in an $18 \times 14 \times 19.5 \mathrm{~cm}$ stimulus compartment. A Futaba FP-S148 servo motor attached to the upper corner of a Masonite panel allowed automatic control of visual access to the female compartment by rotation of the Masonite panel. Wire mesh prevented the male and female birds from making physical contact with each other or with the rotating Masonite panel. Two Christmas tree light bulbs (one red, one green) were centered $11.5 \mathrm{~cm}$ over the window opening, $5 \mathrm{~cm}$ apart, center to center. A $4 \times 32 \times 6 \mathrm{~cm}$ wooden arm was placed on each side of the observation window at floor level and extended into the test arena perpendicular to the window. Infrared photoemitters and detectors were placed $5 \mathrm{~cm}$ apart on each of the arms to detect when the subject was near the window. The subjects easily hopped over the arms while moving about the test arena. The effective area in front of the window monitored by the infrared circuitry was $32 \times 36 \mathrm{~cm}$. All experimental control and data collection were handled by microcomputer.

Procedure. Prior to the start of the experiment, all subjects were given a copulatory pretest. A female quail was placed in the male's home cage for $5 \mathrm{~min}$. Only males that engaged in copulatory behavior during this 5 -min pretest were selected for the experiment. The experiment was run in two replications of 12 subjects each. Each male spent $24 \mathrm{~h}$ of each 48 -h period in the test arena. Two squads of subjects were exchanged between the test arenas and the home cages at midafternoon each day, and daily sessions were run at approximately 1200 and $1600 \mathrm{~h}$. Thus, each subject got alternating midday and late afternoon sessions, but only one session per day.

Male Japanese quail are territorial during the breeding season and experimentally naive subjects can be extremely skittish in the laboratory. They engage in sexual behavior most readily and are most responsive to sexual conditioning if they are thoroughly ha- bituated to the test apparatus and to the experimental procedures. Habituation to a new context is facilitated by time spent in the context and sexual experience obtained there. Therefore, the subjects were allowed to habituate to the test arenas and the changeout schedule for 8 days before the start of the experiment, and on each of these 8 days, a single female was placed in the test arena through the maintenance door to provide a 5 -min copulatory opportunity at the scheduled session time. The subjects were observed until at least one copulation was completed or until 5 min had elapsed. The female was always placed in a different location in the test arena from the location of the observation window that was used during the subsequent conditioning trials.

The subjects were habituated to the stimulus presentation procedures during the next seven scheduled session times. The first, fourth, and seventh habituation sessions consisted of five trials in which the observation window was opened for $2 \mathrm{~min}$. The observation compartment was empty during these sessions. The other four habituation sessions consisted of five trials in which a stimulus light was illuminated for $30 \mathrm{sec}$. The green stimulus light was used during two light-only habituation sessions, and the red stimulus light was used during the other two sessions. Following each habituation session, a female quail was placed in the test arena through the maintenance door to provide the male with a 5-min copulatory opportunity.

Following the habituation phase, first-order conditioning began. Before each session, a female quail was placed in the observation compartment. Stimulus females were randomly assigned to test arenas for each session. The average intertrial interval (ITI) was $10 \mathrm{~min}$ (range, 2-18 min), and each session consisted of five trials. During each trial, visual access to the stimulus female for 2 min served as the US. The first-order CS was provided by the sound and movement of the Masonite panel as it was rotated to provide visual access to the observation compartment. The location of the subject was recorded twice per second during the last $30 \mathrm{sec}$ of the ITI and throughout the US presentation. At the end of the session, the female was removed from the observation compartment and placed in the test arena through the maintenance door. The birds were then allowed a 5-min copulatory opportunity. Daily first-order conditioning trials were conducted for 10 days.

For second-order conditioning, the subjects were assigned to two groups. The subjects in the paired group $(n=12)$ were given three trials per session. Each paired trial consisted of presentation of the CS2 light for $30 \mathrm{sec}$ immediately before the observation window was opened for $30 \mathrm{sec}$ without a female present in the observation compartment. For the subjects in the unpaired group $(n=$ 12), the CS2 presentations and window openings were given on alternating trials. That is, one trial consisted only of a CS2 presentation and the next trial consisted only of a window opening. There were six trials per session, three with CS2 presentations and three with window openings. The average ITI interval remained $10 \mathrm{~min}$, resulting in sessions for the unpaired group that were twice as long as those given the paired group. The red light served as CS2 for half of the subjects, and the green light served as CS2 for the remaining subjects.

The subjects were allowed to copulate with females in the test arenas at the end of each second-order session, as during firstorder conditioning. To slow possible extinction effects, additional first-order conditioning sessions were alternated daily with the second-order conditioning sessions. These first-order sessions were identical to those conducted during initial training, except that three conditioning trials per day were given instead of five. Six second-order conditioning sessions were conducted in all.

\section{Results}

One subject in the unpaired group stopped copulating during first-order conditioning and consistently avoided the observation zone when the window was open. Be- 


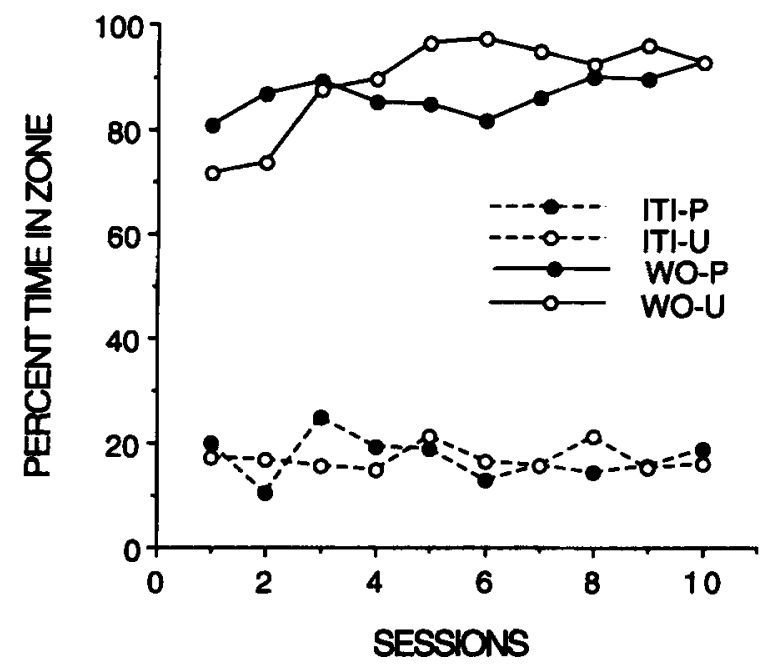

Figure 1. Mean time spent in the observation zone during firstorder conditioning in Experiment 1. ITI-P and IT1-U indicate time in the observation zone during the last 30 sec of the ITI for subjects in the paired and unpaired groups, respectively. WO-P and WO-U indicate time in zone during 2-min window-open periods for subjects in the paired and unpaired groups, respectively.

cause this reduced its exposure to the US, the data from this subject were excluded from all analyses. Figure 1 shows time spent in the observation zone for the 10 firstorder acquisition sessions. The subjects in both groups demonstrated strong approach during window-open periods and spent little time in the observation zone during ITI periods. A three-way repeated measures group $\times$ stimulus (ITI vs. window open) $\times$ session analysis of variance (ANOVA) revealed a significant effect of stimulus $[F(1,21)=355.81, p<.001]$ and a significant stimulus $\times$ session interaction $[F(9,189)=2.23, p<.05]$. The effect of group was not significant $[F<1.0]$.

Figure 2 shows time spent in the observation zone for the last $30 \mathrm{sec}$ of the ITI and for the 30-sec CS2 presentations during the second-order conditioning trials. Quail in the paired group spent more time in the observation zone during CS2 periods than during ITI periods. In contrast, subjects in the rnpaired group did not consistently spend more time in the observation zone during CS2 periods than during ITI periods. In addition, despite the alternation of refresher first-order conditioning sessions with secondorder conditioning sessions, conditioned approach to CS2 began to decline toward ITI baseline levels by the sixth second-order session. A three-way repeated measures group $X$ session $X$ stimulus (ITI vs. CS2) ANOVA of the second-order conditioning data showed significant effects of group $[F(1,21)=7.23, p<.05]$, session $[F(5,105)=2.89$, $p<.05]$, and ITI versus CS2 period $[F(1,21)=10.86, p<$ $.01]$. There was also a significant group $\times$ session $\times$ stimulus (ITI vs. CS2) interaction $[F(5,105)=3.00, p<.05]$.

\section{Discussion}

Responding to the second-order visual CS developed in the paired group but not in the unpaired group. These results indicate that effectiveness of the second-order stimulus in eliciting approach depended on pairings of CS2 with the first-order conditioned stimulus.

\section{EXPERIMENT 2}

The necessity of CS1-CS2 pairings for second-order conditioning was demonstrated in Experiment 1. Only the group given paired second-order trials developed conditioned approach to CS2. Experiment 2 was designed to test the necessity of CS1-US pairings for establishing responding to $\mathrm{CS} 2$. One group received paired first-order trials and a second group received unpaired first-order trials. Then, both groups were given paired second-order trials.

\section{Method}

Subjects. Twelve adult males raised under conditions identical to those of Experiment 1 served as subjects. They were approximately 4 months old at the start of the experiment. Six had previously served in an experiment on copulation with taxidermic models; the remaining 6 were sexually naive. Twelve adult sexually receptive females served as stimulus females and copulation partners.

Apparatus. The same apparatus was used as in Experiment 1. To provide visual exposure to female quail for subjects in the unpaired group, cylinders made of $2.5-\mathrm{cm}$ wire mesh were affixed with spring clips to the center of the wire mesh maintenance doors, slightly above the level of the arena floor.

Procedure. Pretesting and habituation procedures were conducted as in Experiment 1. Prior to first-order conditioning, subjects were assigned to two groups, with group assignment balanced for prior experience. Subjects in the paired group $(n=6)$ were given five first-order conditioning trials per session, as in Experiment 1. Subjects in the unpaired group $(n=6)$ were given similar first-order trials, but without a female in the observation compartment. Instead,

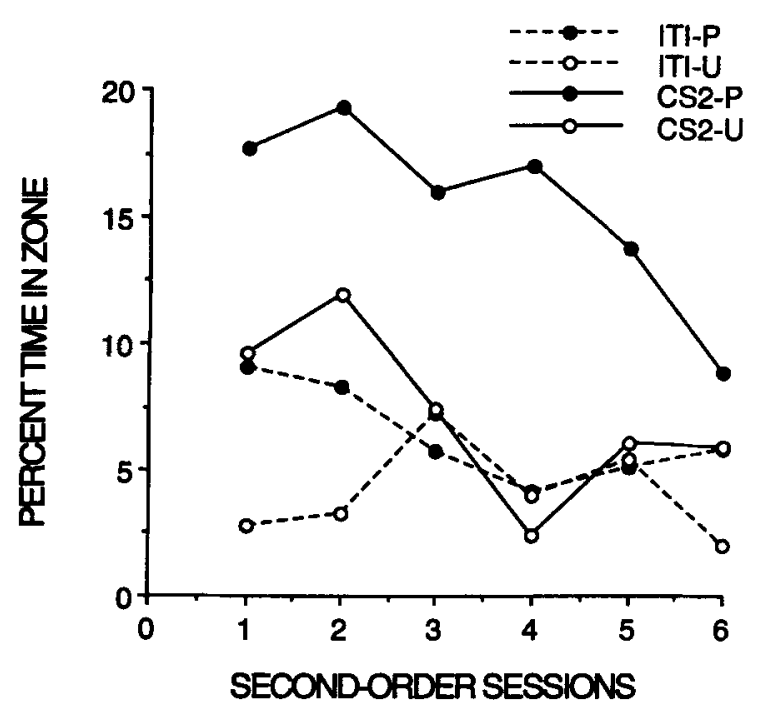

Figure 2. Mean time spent in the observation zone during secondorder conditioning in Experiment 1. ITI-P and ITI-U indicate time in the observation zone during the last $30 \mathrm{sec}$ of the ITI for subjects in the paired and unpaired groups, respectively. CS2-P and CS2-U indicate time in zone during 30-sec CS2 periods for subjects in the paired and unpaired groups, respectively. 
visual exposure to a female quail was provided for them for $5 \mathrm{~min}$ before and after each session. Thus, the unpaired group received the same total duration of visual exposure to females during each firstorder session as the paired group. Visual exposure to females for subjects in the unpaired group was provided by placing a female in the wire cylinder clipped to the cage maintenance door. First-order conditioning was conducted for 10 sessions.

All subjects were then given paired second-order conditioning trials, as in the paired group of Experiment 1. As in Experiment 1, additional first-order conditioning sessions were alternated with the second-order conditioning sessions. Six second-order conditioning sessions were conducted. All sessions during the second phase of the experiment consisted of three trials. Subjects in the unpaired group were given $3 \mathrm{~min}$ instead of $5 \mathrm{~min}$ of visual exposure to a female quail before and after the additional first-order conditioning sessions so that their total exposure to females would be the same as the total exposure received by subjects in the paired group.

\section{Results}

Figure 3 shows time spent in the observation zone during the first-order conditioning phase for the two groups. The subjects in the paired group showed clear differentiation between ITI and window-open periods. In contrast, the subjects in the unpaired group responded at similar low levels during the ITI and window-open periods. These effects were confirmed by a three-way repeated measures group $\times$ stimulus (ITI vs. window open) $\times$ session ANOVA on the 10 first-order conditioning sessions. There were significant main effects of group $[F(1,10)=$ $48.41, p<.001]$ and stimulus $[F(1,10)=254.61, p<$ $.001]$. The main effect of session was nonsignificant $[F(9,90)=1.74, p>.05]$, but the group $\times$ stimulus interaction was significant $[F(1,10)=145.53, p<.001]$.

Figure 4 shows the second-order data for subjects in the two groups. Quail in the paired group showed clear discrimination between ITI and CS2 periods during the

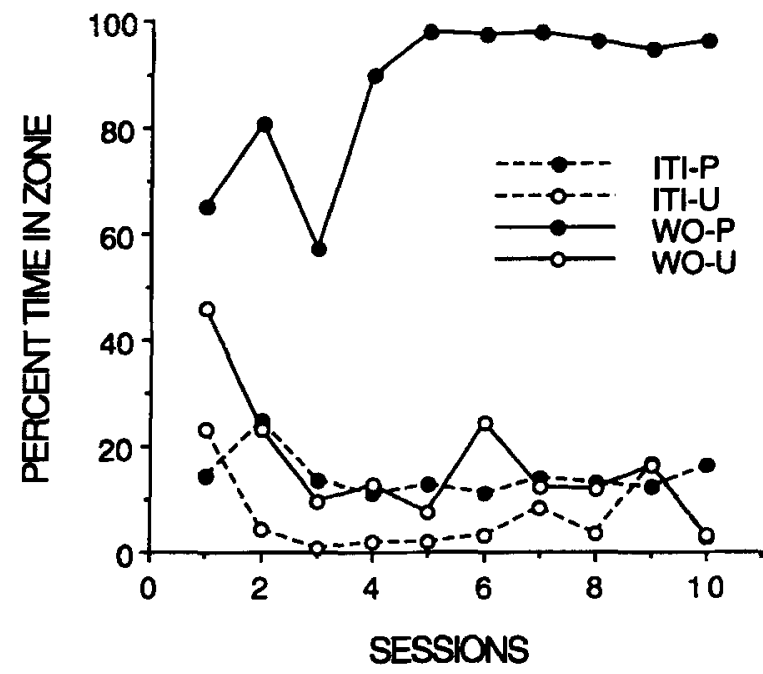

Figure 3. Mean time spent in the observation zone during firstorder conditioning in Experiment 2. ITI-P and ITI-U indicate time in the observation zone during the last 30 sec of the ITI for subjects in the paired and unpaired groups, respectively. WO-P and WO-U indicate time in zone during 2 -min window-open periods for subjects in the paired and unpaired groups, respectively.

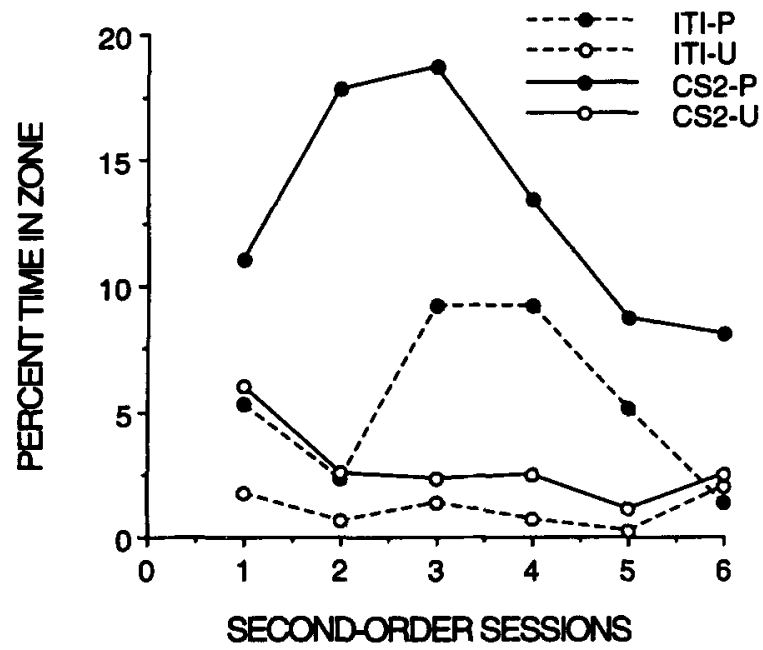

Figure 4. Mean time spent in the observation zone during secondorder conditioning in Experiment 2. ITI-P and ITI-U indicate time in the observation zone during the last 30 sec of the ITI for subjects in the paired and unpaired groups, respectively. CS2-P and CS2-U indicate time in zone during 30-sec CS2 periods for subjects in the paired and unpaired groups, respectively.

first few second-order conditioning sessions. In contrast, subjects in the unpaired group did not discriminate between ITI and CS2 periods. As in Experiment 1, secondorder conditioning declined with continued training.

A three-way repeated measures group $\times$ stimulus (ITI vs. CS2) $\times$ session ANOVA on the first two sessions of second-order conditioning revealed a significant main effect of stimulus (ITI vs. CS2) $[F(1,10)=6.78, p<$ $.05]$. The triple interaction of group $\times$ stimulus $\times$ session was also significant $[F(1,10)=7.87, p<.05]$. This interaction indicates that the higher level of responding observed during CS2 than during the ITI significantly depended on group assignment across second-order training sessions.

A comparable analysis that included data for all six second-order sessions revealed significant main effects of group $[F(1,10)=6.53, p<.05]$ and stimulus (ITI versus CS2 $)[F(1,10])=6.70, p<.05]$. A significant group $\times$ session interaction was also obtained $[F(5,50)=$ $2.58, p<.05]$.

\section{Discussion}

The subjects in the paired group acquired clear firstand second-order conditioning. In contrast, the subjects in the unpaired group did not acquire either first- or second-order conditioned responding. These results indicate that prior successful first-order conditioning is necessary for the development of responding to the second-order stimulus.

\section{EXPERIMENT 3}

Experiments 1 and 2 demonstrated that both first-order pairings and second-order pairings are necessary for the 
acquisition of conditioned responding to the secondorder stimulus. Experiment 3 was conducted to determine whether continued responding to the second-order stimulus depends on maintaining the integrity of the firstorder conditioned stimulus. After second-order conditioning, responding to CS1 was extinguished for half the subjects. All the birds were then tested for their responding to CS2.

\section{Method}

Subjects. Twenty-four sexually naive adult male quail, approximately 5-8 months old at the beginning of the experiment, served as subjects. All were raised as described in Experiment 1. Twentyfour adult sexually receptive female quail served as stimulus females and copulation partners.

Apparatus. The same apparatus as described in Experiment 1 was used.

Procedure. The experiment was conducted in two replications of 12 birds each. Pretesting, habituation procedures, and first-order conditioning sessions were conducted as in Experiment 1. After the completion of first-order conditioning, two sessions of secondorder conditioning were conducted, separated by an additional session of first-order conditioning. Two trials were given during each second-order session.

Following the first two second-order conditioning sessions, subjects were assigned to either an extinction group $(n=12)$ or a control group $(n=12)$. Subjects in the extinction group were given daily first-order extinction sessions. Each extinction trial consisted of the opening of the observation window for $2 \mathrm{~min}$, with no female present in the observation chamber. Five extinction trials were conducted per session for eight sessions. Subjects in the control group continued to be moved between the home cages and the test arenas, but were given no training sessions during this time. All subjects continued to receive daily copulatory opportunities with females in the test chambers. These were provided after the daily sessions for the extinction group.

After the extinction phase, all subjects were again given two second-order conditioning sessions, separated by an additional first-order conditioning session. As in the initial second-order phase, two conditioning trials per session were given during the final second-order conditioning phase.

\section{Results and Discussion}

Two subjects in the control group showed minimal approach to the US during first-order conditioning. Therefore, their data were excluded from all analyses. The remaining subjects in both groups showed strong approach to the visible female during first-order conditioning, as in Experiments 1 and 2. This was confirmed by a threeway repeated measures group $X$ stimulus (ITI vs. US period) $\times$ session ANOVA. Subjects in both groups spent significantly more time in the observation zone during US periods than during ITI periods $[F(1,20)=358.94$, $p<.001$.

The time subjects spent in the observation zone when the observation window was open dropped rapidly during extinction sessions for the extinction group, leveling off at approximately $20 \%$ of the first-order acquisition asymptote.

Figure 5 shows the second-order conditioning data for subjects in both groups. Both groups showed approach to the second-order conditioned stimulus during the first

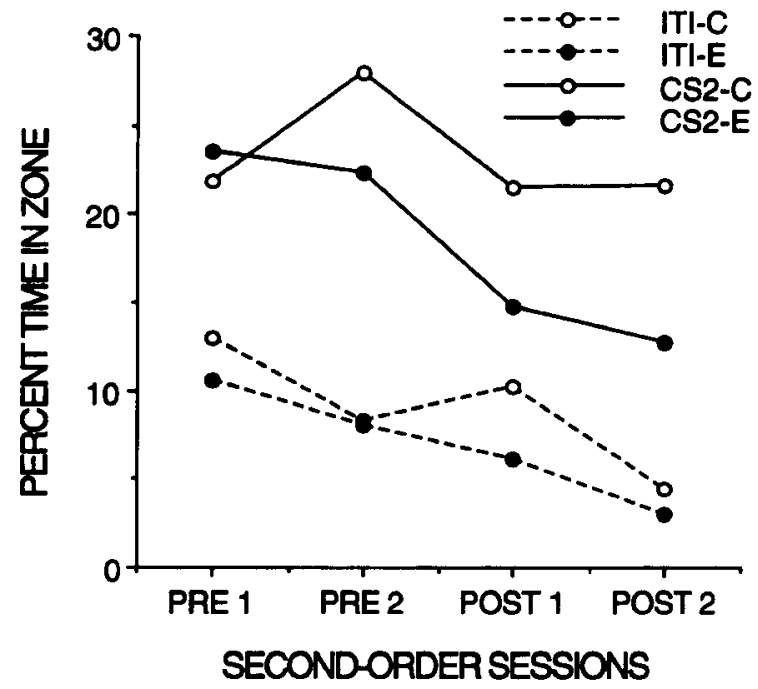

Figure 5. Mean time spent in the observation zone during secondorder conditioning in Experiment 3. ITI-C and ITI-E indicate time in the observation zone during the last $30 \mathrm{sec}$ of the ITI for subjects in the control and extinction groups, respectively. CS2-C and CS2-E indicate time in zone during 30-sec CS2 periods for subjects in the control and extinction groups, respectively.

two second-order sessions, prior to the first-order extinction manipulation. A clear separation in the time that the subjects spent in the observation zone during CS2 as opposed to the ITI remained after extinction of first-order stimulus. A four-way repeated measures group $\times$ stimulus (ITI vs. CS2 period) $\times$ phase (pre- versus postextinction) $\times$ session ANOVA revealed significant effects of stimulus $[F(1,20)=13.25, p<.01]$ and phase $[F(3,60])=13.53, p<.01]$. No other effects were significant (all $F \mathrm{~s}<1$ ). Similar results were obtained with an analysis of the data from the last preextinction session and the first postextinction session.

If extinction of responding to CS1 had influenced responding to CS2, a significant stimulus $\times$ phase $\times$ group interaction would have been obtained. The failure to observe this interaction effect indicates that extinction of the first-order conditioned stimulus did not weaken responding to CS2. The persistence of second-order responding despite extinction of responding to CS1 indicates that second-order responding was not mediated by the status of the first-order conditioned stimulus.

\section{EXPERIMENT 4}

In Experiment 3, devaluation of CS1 by extinction of first-order responding did not produce a significant change in responding to CS2. Experiment 4 was conducted to determine whether devaluation of the US would affect second-order conditioned responding. For male subjects, devaluation of a sexual US can be accomplished by reducing circulating levels of testosterone (Holloway \& Domjan, 1993b) or by sexual satiation (Hilliard \& Domjan, in press). Both methods have been found to attenu- 
ate first-order sexual conditioned responding. In Experiment 4 , the effects of sexual satiation on second-order conditioned responding were investigated.

\section{Method}

Subjects. The subjects were 12 experimentally naive male Japanese quail. All were hatched and raised under conditions similar to those described in Experiment 1 and were 5-6 months old at the start of the habituation phase. The subjects were originally scheduled for use in another experiment and spent alternate days in test arenas different from those used in Experiment 4 over a 34day period. In addition, they received 5-min copulatory opportunities on six occasions. However, they did not receive any explicit conditioning trials before Experiment 4 . Six sexually receptive adult female Japanese quail served as stimulus females and copulation partners.

Apparatus. The same six test arenas used in Experiments 1, 2, and 3 were used.

Procedure. As in previous experiments, the subjects spent $24 \mathrm{~h}$ of each 48-h period in the test arenas, and all of them were given a training session every day, half before the daily tradeout and half after the tradeout. On each of the first 4 days, the subjects were given a 5 -min copulatory opportunity with a sexually receptive female in the test arenas. During the next 5 days, they were given sessions to habituate them to the stimuli to be used in the experiment. In all habituation sessions, the average intertrial interval was $10 \mathrm{~min}$. Three habituation sessions consisted of five trials each in which the observation window was opened for $2 \mathrm{~min}$, revealing an empty observation compartment. Two habituation sessions were light-only sessions and consisted of five 30 -sec light presentations. In three of the test arenas, the red light was used, and in the other three test arenas the green light was used. At the end of each habituation session, the males were given a 5-min copulatory opportunity with a sexually receptive female.

Following the habituation phase, the subjects were given 8 days of first-order conditioning, with sessions conducted as in Experiment 1. After first-order conditioning, the subjects were given two second-order conditioning sessions, each of which consisted of three paired trials. The observation window was opened after each CS2 presentation and remained open for $2 \mathrm{~min}$, allowing the subjects to see into the empty observation compartment. The two second-order sessions were separated by a first-order conditioning session consisting of three trials.

All subjects were then tested for approach to the second-order light CS2 under both US devaluation and nondevaluation conditions. Test sessions were conducted in the same manner as secondorder conditioning sessions. The value of the US was manipulated by changing the subjects' level of sexual deprivation. The US devaluation test was conducted around noon, after the subjects had been left in the test arenas overnight with 2 females. In addition, just before the US devaluation test session, 5 additional females were added to each test arena, 1 female at a time, at 15 -min intervals. After the last female had been in the test arena for $15 \mathrm{~min}$, all the females were removed, and the test session was started. The US nondevaluation test was conducted after the subjects had been sexually deprived for at least $24 \mathrm{~h}$. US devaluation and nondevaluation sessions were conducted in a counterbalanced order, separated by a first-order refresher conditioning session.

\section{Results and Discussion}

One subject failed to show any approach to the visible female throughout first-order training, so its data were excluded from all analyses. As in Experiments 1, 2, and 3 , subjects spent over $90 \%$ of the window-open periods in the observation zone during the first-order conditioning phase. A repeated measures stimulus $\times$ session

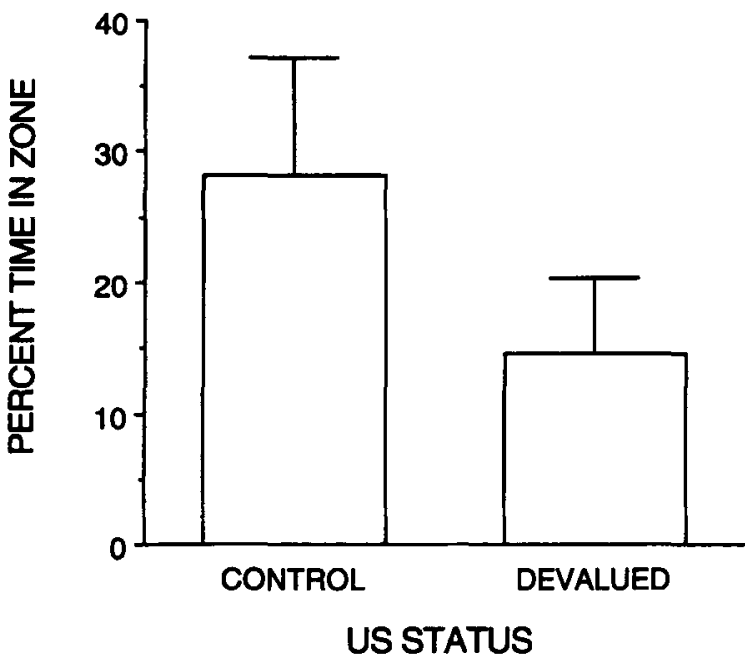

Figure 6. Mean time spent in the observation zone during CS2 presentations in both a normal second-order conditioning session (control) and a second-order conditioning session after sexual satiation (US devalued).

ANOVA revealed significantly more approach during window-open periods than during intertrial intervals $[F(1,10)=224.47, p<.001]$.

The US devaluation manipulation successfully lowered the sexual motivation of the subjects. This was evident from the proportion of the time that the subjects spent in the observation zone when the window was open during the test sessions. Subjects spent significantly more of their time near the open window when they were sexually deprived (mean $=46 \%$ ) than when they were sexually satiated $($ mean $=20 \%)[F(1,10)=15.76, p<.001]$.

Figure 6 shows approach to the light CS2 during the US devaluation and control test sessions. Significantly less approach to CS2 was evident during the US devaluation session than during the control session $[F(1,10)=$ $6.40, p<.05]$. These results indicate that US devaluation reduced, but did not eliminate, approach to a second-order CS for visual exposure to a female.

During the test sessions, presentations of CS2 continued to be followed by CS1. One might suggest that the reduction in responding to CS2 that occurred with US devaluation was mediated by reduced responding to CS1 produced by sexual satiation. However, this seems unlikely, considering that substantial reductions in responding to CS1 had no significant effect on responding to CS2 in Experiment 3. Therefore, the effects of US devaluation on second-order conditioned responding probably were not mediated by changes in CS1.

\section{GENERAL DISCUSSION}

The results of the present experiments demonstrate sexual second-order conditioning in male Japanese quail. Conditioned responding to the second-order stimulus did not develop if CS2 was not paired with CS1 (Experiment 1) or if CS1 was not paired with the US (Experi- 
ment 2). Once second-order responding was established, it was independent of subsequent extinction of responding to CS1 (Experiment 3 ) but was attenuated by reduced sexual motivation (Experiment 4).

Zamble et al. (1985) previously demonstrated secondorder sexual conditioning in experiments with male rats and also reported that after acquisition, second-order conditioning is independent of the integrity of the firstorder stimulus. The present data are consistent with these findings and extend their generality in several ways. First, the results were obtained with an avian rather than a rodent species. Second, conditioning was evident in behavior directed toward CS2 rather than in the facilitation of copulation with a female. Third, the present experiments included a wider range of postconditioning manipulations to evaluate the autonomy of second-order sexual conditioning.

Postconditioning manipulations have been of particular interest in studies of second-order conditioning because they can shed light on the nature of the learning. Early results suggested that second-order conditioning might be fundamentally different from first-order conditioning. In particular, second-order conditioning appeared to be immune to various postconditioning manipulations that strongly influenced first-order conditioning.

Reduction in the value of the US (through counterconditioning, habituation, or reduced motivation) was found to attenuate responding to CS1 in fear and appetitive conditioning in rats and pigeons (e.g., Holland \& Rescorla, 1975a; Rescorla, 1973), conditioned analgesia in rats (Ross, 1988), and sexual conditioning in quail (Holloway \& Domjan, 1993b). In contrast, a comparable manipulation following second-order conditioning (extinction of responding to $\mathrm{CS} 1$ ) did not attenuate second-order responding in studies of fear conditioning in rats (Helmstetter \& Fanselow, 1989; Rizley \& Rescorla, 1972) and appetitive conditioning in rats (Holland \& Rescorla, 1975b). Postconditioning devaluation of the US also did not disrupt second-order responding in fear conditioning and appetitive conditioning in rats (Holland \& Rescorla, 1975a; Rescorla, 1973). These results encouraged the interpretation that, in contrast to first-order conditioning, second-order conditioning involved a direct association of CS2 with the conditioned response. Presumably, this $\mathrm{S}-\mathrm{R}$ learning made second-order conditioning immune to postconditioning changes in either CS1 or the US.

Subsequent studies have raised questions about the generality of the S-R interpretation of second-order conditioning. Rashotte, Griffin, and Sisk (1977), using an autoshaping procedure with pigeons, found attenuated second-order responding after extinction of responding to CS1. Further investigation revealed that the nature of the stimuli used as CS1 and CS2, as well as the temporal relation between $\mathrm{CS} 1$ and $\mathrm{CS} 2$, were important factors in determining whether or not second-order conditioning involves S-R learning (e.g., Rescorla, 1979, 1980, 1982). However, the effect of US devaluation was not explored in these studies.
The particular pattern of findings obtained in the present experiments has not been commonly observed in studies of second-order conditioning. We found two precedents in the literature, however. In a study of electrodermal conditioning in humans, Davey and McKenna (1983) found that responding to CS2 did not decrease after extinction of responding to CS1 but decreased after US devaluation. In experiments on conditioned analgesia in rats, Ross (1988) also found that responding to CS2 was not influenced by extinction of CS1 but was reduced by counterconditioning of the shock US (by pairing the shock with morphine).

Instances of second-order conditioned responding that are reduced by US devaluation but not by extinction of CS1 may be mediated by an association between representations of CS2 and of the US. Such an association could be established if CS1 activates a representation of the US during second-order trials because of its prior conditioning history. CS2 could become associated with this activated US representation.

According to the preceding scenario, a CS2--US association will be established in second-order conditioning only in situations where first-order conditioning results in a CS1-US association. Therefore, in every case where US devaluation attenuates second-order responding, US devaluation should also attenuate conditioned responding to CS1. The available evidence is consistent with this requirement. All the instances in which US devaluation has been found to reduce second-order responding that we are aware of (Davey \& McKenna, 1983; Ross, 1988; and Experiment 4 of the present report) involve preparations in which US devaluation also reduces responding to CS1.

It is important to note that the converse prediction is not implied by the mechanism that we have proposed. That is, establishment of a CS1-US association in firstorder conditioning does not guarantee that CS2 will become associated with the representation of the US that is activated by $\mathrm{CS} 1$ during second-order conditioning trials. For example, first-order fear conditioning in rats results in a CS1-US association, but second-order conditioning does not result in a CS2-US association (e.g., Rescorla, 1973).

The present findings, together with the available literature, leave several important questions about secondorder conditioning unanswered. One question is why a CS2-US association does not result from CS2-CS1 pairings in all of the various instances in which CS1 activates a representation of the US. Perhaps CS2-CS1 pairings result in a CS2-US association only if CS1 activates a sufficiently detailed representation of the US. A second important question is why in some situations secondorder conditioning results in a CS2-US association with no evidence of the formation of a CS2-CS1 association. In the present studies, as well as in GSR conditioning (Davey \& McKenna, 1983), second-order conditioning seemed to result in a CS2-US association, but there was no evidence of the formation of a CS2-CS1 association. 


\section{REFERENCES}

Akins, C. K., Domjan, M., \& GutiéRrez, G. (1994). Topography of sexually conditioned behavior in male Japanese quail (Coturnix japonica) depends on the CS-US interval. Journal of Experimental Psychology: Animal Behavior Processes, 20, 199-209.

Crawford, L. L., Akins, C. K., \& Domjan, M. (1994). Stimulus control of copulatory behavior in sexually naive male Japanese quail (Coturnix japonica): Effects of test context and stimulus movement. Journal of Comparative Psychology, 108, 252-261.

Crawford, L. L., \& Domjan, M. (1993). Sexual approach conditioning: Omission contingency tests. Animal Learning \& Behavior, 21, 42-50.

Davey, G. C. L., \& McKenna, I. (1983). The effect of postconditioning revaluation of CS1 and UCS following Pavlovian second-order electrodermal conditioning in humans. Quarterly Journal of Experimental Psychology, 35B, 125-133.

Domjan, M. (1994). Formulation of a behavior system for sexual conditioning. Psychonomic Bulletin \& Review, 1, 421-428.

Domjan, M., Greene, P., \& North, N. C. (1989). Contextual conditioning and the control of copulatory behavior by species-typical sign stimuli in male Japanese quail. Journal of Experimental Psychology: Animal Behavior Processes, 15, 147-153.

Domjan, M., \& HALL, S. (1986). Determinants of social proximity in Japanese quail (Coturnix coturnix japonica): Male behavior. Journal of Comparative Psychology, 100, 59-67.

Helmstetter, F. J., \& FAnselow, M. S. (1989). Differential secondorder aversive conditioning using contextual stimuli. Animal Learning \& Behavior, 17, 205-212.

Hilliard, S., \& Domjan, M. (in press). Effects on sexual conditioned behavior of devaluing the US through satiation. Quarterly Journal of Experimental Psychology.

Holland, P. C., \& Rescorla, R. A. (1975a). The effect of two ways of devaluing the unconditioned stimulus after first- and second-order appetitive conditioning. Journal of Experimental Psychology: Animal Behavior Processes, 1, 355-363.

- Holland, P. C., \& Rescorla, R. A. (1975b). Second-order condition- ing with food unconditioned stimulus. Journal of Comparative \& Physiological Psychology, 88, 459-467.

Holloway, K. S., \& Domjan, M. (1993a). Sexual approach conditioning: Unconditioned stimulus factors. Journal of Experimental Psychology: Animal Behavior Processes, 19, 38-46.

Holloway, K. S., \& Domjan, M. (1993b). Sexual approach conditioning: Tests of unconditioned stimulus devaluation using hormone manipulations. Journal of Experimental Psychology: Animal Behavior Processes, 19, 47-55.

Rashotte, M. E., Griffin, R. W., \& Sisk, C. L. (1977). Second-order conditioning of the pigeon's keypeck. Animal Learning \& Behavior, 5, 25-38.

RESCORLA, R. A. (1973). Effect of US habituation following conditioning. Journal of Comparative \& Physiological Psychology, 83, 137-143.

ResCORLA, R. A. (1979). Aspects of the reinforcer learned in secondorder Pavlovian conditioning. Journal of Experimental Psychology: Animal Behavior Processes, 5, 79-95.

Rescorla, R. A. (1980). Pavlovian second-order conditioning: Studies in associative learning. Hillsdale, $\mathrm{NJ}$ : Erlbaum.

RESCORLA, R. A. (1982). Simultaneous second-order conditioning produces S-S learning in conditioned suppression. Journal of Experimental Psychology: Animal Behavior Processes, 8, 23-32.

Rizley, R. C., \& Rescorla, R. A. (1972). Associations in secondorder conditioning and sensory preconditioning. Journal of Comparative \& Physiological Psychology, 81, 1-11.

Ross, R. T. (1988). Pavlovian second-order conditioned analgesia. Journal of Experimental Psychology: Animal Behavior Processes. 12, 32-39.

Zamble, E., Hadad, G. M., Mitchell, J. B., \& Cutmore, T. R. H. (1985). Pavlovian conditioning of sexual arousal: First- and secondorder effects. Journal of Experimental Psychology: Animal Behavior Processes, 11, 598-610.

(Manuscript received March 29, 1994; revision accepted for publication September $1,1994$. 